\title{
DEVELOPMENT OF A M3S PROTOTYPE SYSTEM USING DIGITAL SIGNAL PROCESSOR
}

\author{
Jen-Chien Chien', Meng-Lun HSueh', Chien-Chi Chen', Jer-Junn LuH ${ }^{2}$, JN-Shin Lal ${ }^{2}$, \\ FOK-CHING CHONG'
}

'Institute of Electrical Engineering, National Taiwan University, Taipei

${ }^{2}$ Department of Physical Medicine and Rehabilitation, National Taiwan University Hospital,

Taipei, Taiwan

\section{ABSTRACT}

Acute disabilities always need different types of assistive devices in their daily life. Usually, each of these devices is designed for only one particular usage. Because of this, an acute disable may have to use a joystick to manipulate his wheelchair, used a mouse to control computer, or used a keyboard to control movement of mechanical arm. Thus no consideration of interrelations among these tools was discussed.

In 1990, the technical working committee 173 (TC-173/sc-1/wg-7) of ISO (International Organization for Standardization) using serial interface protocol (ISO 7176-7) to integrate all types of assistive tools for the disabilities. At the same time, the European Common Commission funded the research for M3S interface (Multiple Masters Multiple Slave). It provides the disable a concrete integral control for his movement, working, environment control and communication. This is an integrated intelligent real time control capability protocol. It is a plug and play device interface.

We design and develop a M3S protocol using TMS320F243 DSP chip. The system was tested for I/O control and it meets the equipments supported by the M3S working group. Thus, it would bring lots of comforts for the disable.

Biomed Eng Appl Basis Comm, 2003(December); 15: 212-216.

Keywords: intelligent protocol, assistive devices, M3S

\section{INTRODUCTION}

Acute disabilities always need different types of assistive devices like molor wheelchair, environmental control system and computer in their daily life... These assistive devices are manufactured by various research units and manufacturers and no consideration of

Received: Oct 9, 2003; Accepted: Nov 6, 2003

Correspondence: Jen-Chien Chien,

Room 341, Institute of Electrical Engineering, National Taiwan University, No.1, Sec.4, Roosevelt Road, Taipei, Taiwan 106

E-mail: janelove@pchome.com.tw interrelation among these tools were taken into account. Therefore, if we integrate these assistive devices into one system, we can reduce usage complexity. It can be more convenience for users. The M3S is a plug and play device interface and once plugin, the new device will integrate and communicate with existing devices in use.

According to the M3S standard the protocol is divided into three items, the input and output item, the intermediate item and the control \& configuration unit (Figure 1). This include the integrated mechanism of the systemic resource, the safety monitored mechanism. These are connected to a common serial bus, the $M 3 \mathrm{~S}$ bus and all operations in this system are based on this bus.

In the transmission control signal phase, the user 
can use one input device such as keyboard, or mouse to control the various output device such as wheelchair, or manipulator.

In the safety monitored phase, the system monitor the overall system safety and acknowledge safety should receive confirmation from all devices. In the integrated mechanism of the systemic resource phase, the control \& configuration module (CCM) maintain the distributed configuration information the devices. This system configuration describes the setup of the complete system and all possible actions for the special needs of a user.

\section{METHOD}

The M3S specification is described in a formal document by ISO/TC-173/SC-1/WG-7. The M3S standard generalizes the OSI reference model to three layers: the application program layer, the software

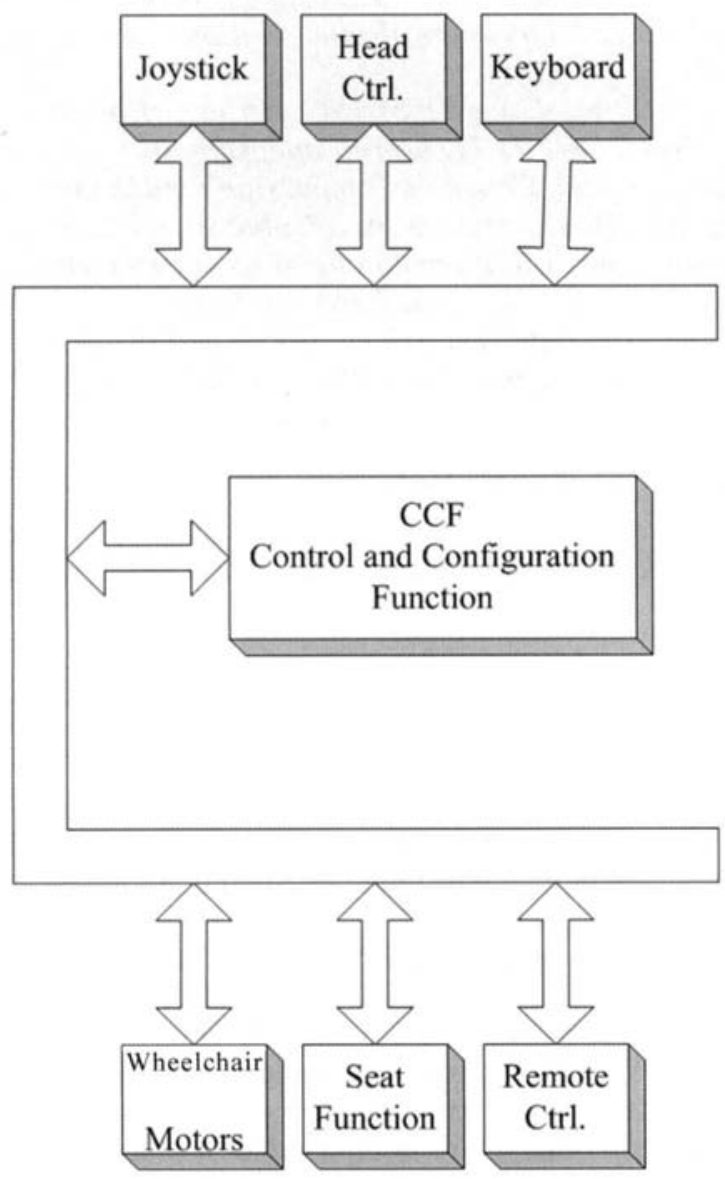

Fig. 1 the M3S standard

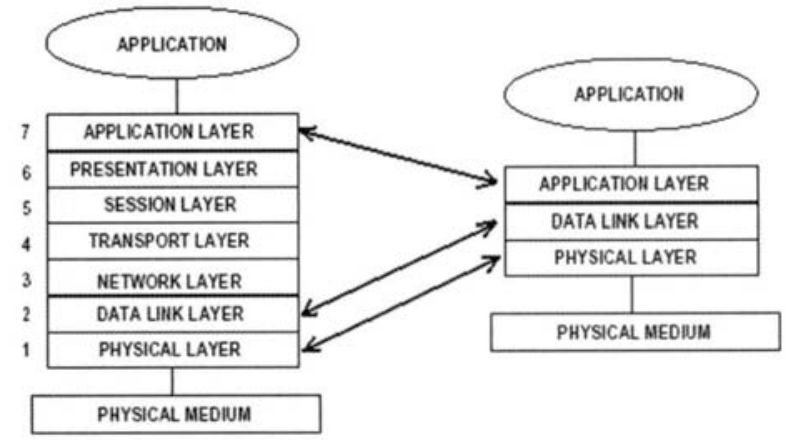

Fig. 2 Relationship of OSI to M3S

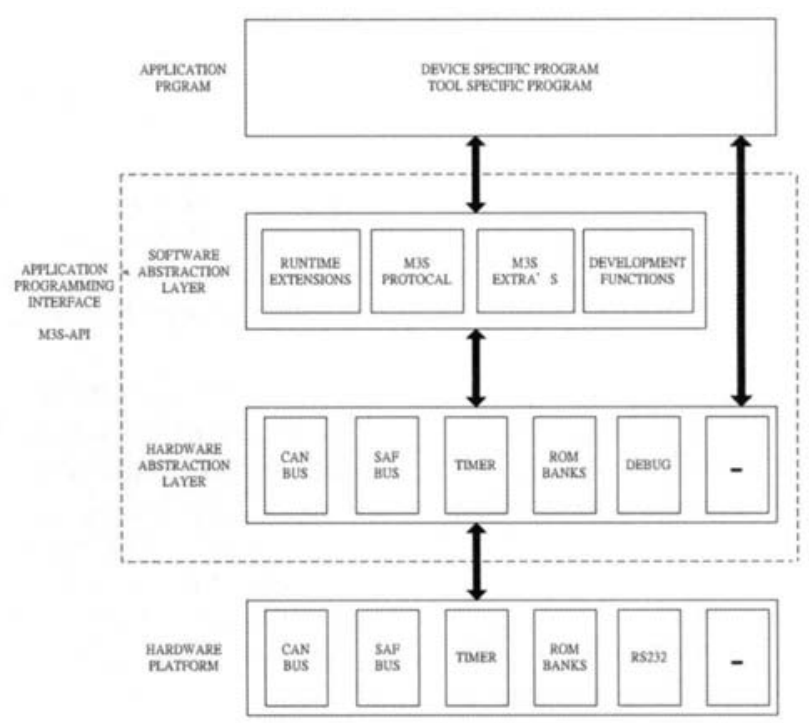

Fig. 3 The M3S standard architecture

abstraction layer and the hardware layer (Figure 2 and Figure 3).

A M3S system is based on a bus and devices. The bus contains three parts: 2 lines for digital communication (CAN bus), 2 lines for power distribution (POW bus) and 2 lines for safety features (SAF bus) (Figure 4). Devices are connected to this bus. Some devices on the bus can link the local bus to one or more remote bus parts in the M3S system using a (wireless) link.

The Control Area Network bus bus (CAN) provides a high safe standard of the serial transmission control protocol and the transmitted rate can reach $1 \mathrm{Mbits} / \mathrm{sec}$. Through broadcasting, the CAN bus can receive the message when the identifier of the installation and the identifier of the inner message are the same. This communication standard has extensive error detection and error recovery facilities. 
In order to further increase the user's safety, two additional items are included in the M3S-system: the key switch and the dead man switch. The key switch can be operated by the user or a helper to turn the system on or off. The dead man switch allows the user to halt these devices safely and quickly by simply releasing it.

The digital circuit of the input device collect the digital and analog signals into the F243 DSP CPU through the GPIO or analog-digital converter. And then the F243 DSP CPU converts the collected signal into the digital signal to the M3S bus (Figure 5).

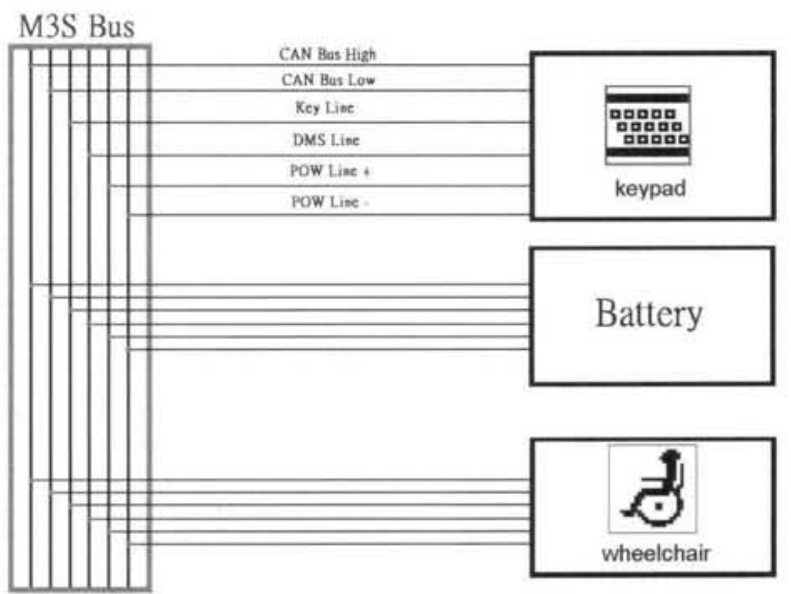

Fig. 4 Three parts of the M3S BUS

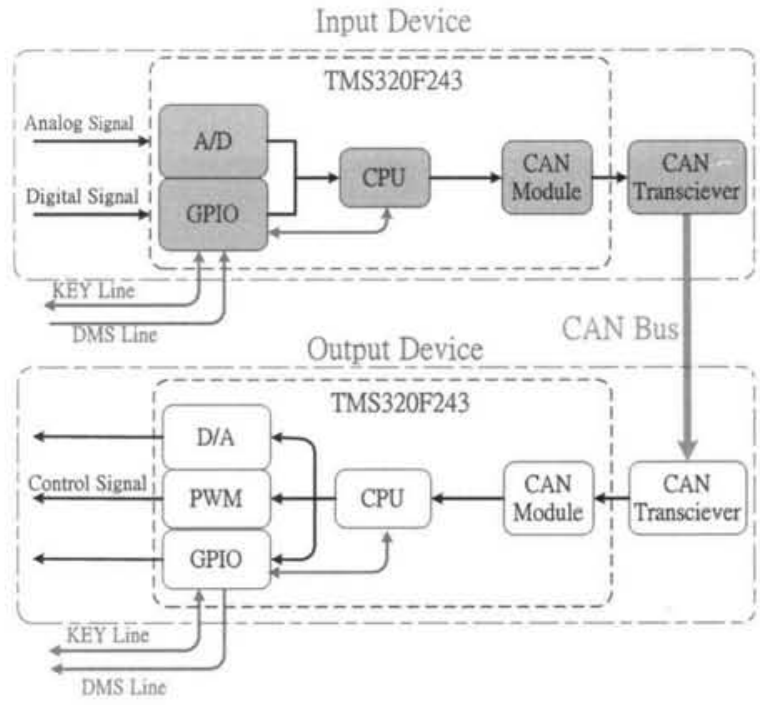

Fig. 5 the diagram of the input and output device
The kernel circuit in this system is the TMS320F243 Evaluation Module (EVM).

\section{IMPLEMENTATION}

\subsection{Software}

In our system the firmware is the TI C2000 Code Composer ${ }^{\mathrm{TM}}$ and the VisSim/Embedded Controls Developer ${ }^{\mathrm{TM}}$.

Figure6 is the algorithm for the input device:

* Analog control signal is sampled by the analog digital converter (ADC) or digital control signal is sampled by the GPIO.

* The F243 DSP CPU codes the sampled signal according to the CAN transmitted protocol.

* The GPIO that is connected with the SAF Bus simultaneously detect the safe monitoring signal.

* If the GPIO doesn't receive the safe monitoring signal, the GPIO transmits the signal that is coded by the CAN module to the CAN BUS.

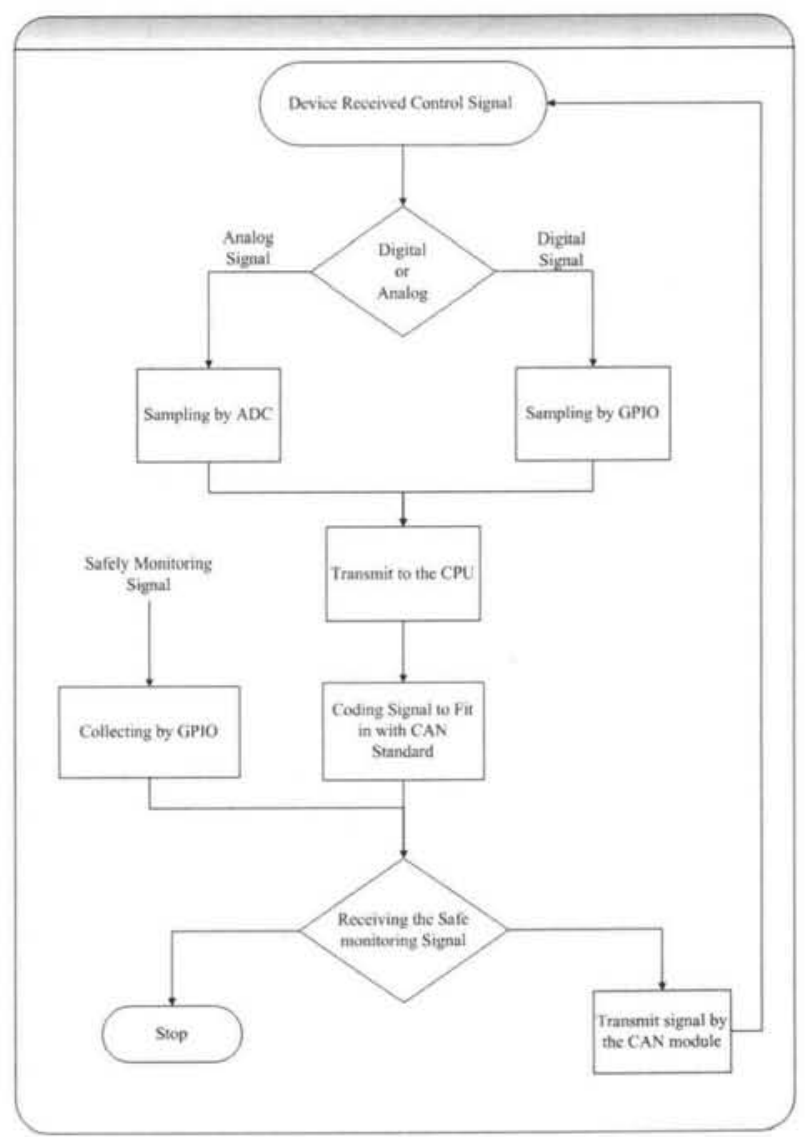

Fig.6 Algorithm for the input device 


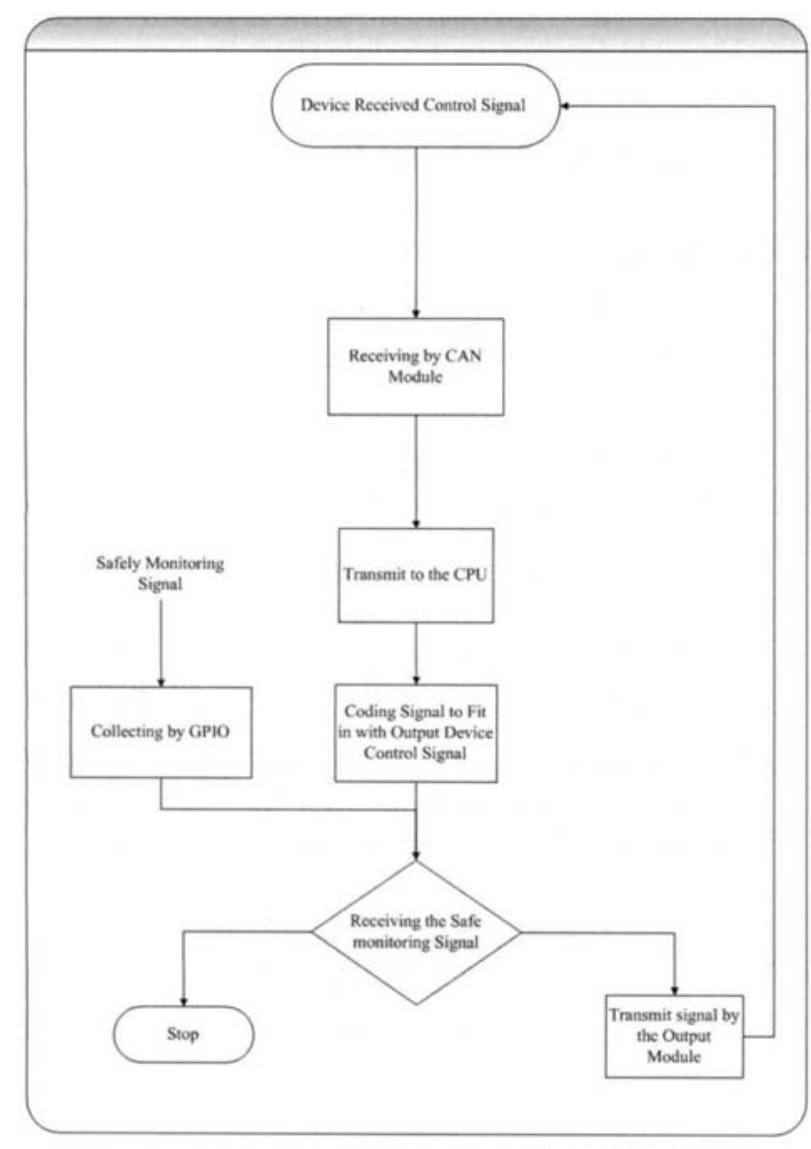

Fig. 7 Algorithm for the output device

* If the GPOI receives the safe monitoring signal, it stop transmitting the signal to the CAN BUS.

Figure 7 is the algorithm for the input device:

* The controlled signal is received by the CAN BUS through the CAN Module.

* The F243 DSP CPU codes the sampled signal according to the output control signal protocol.

* The GPIO that is connected with the SAF Bus simultaneously detect the safe monitoring signal.

* If the GPIO doesn't receive the safe monitoring signal, the GPIO transmits the output signal that is coded by the CAN module to the CAN BUS.

* If the GPOI receives the safe monitoring signal, it stop transmitting the signal to the CAN BUS.

\subsection{Hardware}

The M3S BUS prototype chart is showed in figure 8. The left dotted block is the input apparatus and the right dotted block is the output apparatus. The input devices are composed of the control interface and the digital circuit. The output devices are composed of the
Effect-Device interface and the digital circuit by the same token. In order to ensure the safety in this system, we can use the firmware to control the signals.

The figure 9 is the M3S prototype system. The left block shows the input devices that we design and the right block shows the output devices. The SAF BUS connects the input and the output devices through the GPIO and the CAN BUS transmits the controlled signals.

\section{RESULT}

Our prototype consists of three inputs and three outputs. Figure 10 are the test results for the simulated three controlled directional signals of the wheelchair motor and one "KEY Line" signal. The $x$-axis is the actual test time that the unit is second. The $y$-axis is the signal amplitude that the unit is 5 voltages. The labeled 1 , labeled 2 and labeled 3 are individual "Forward",

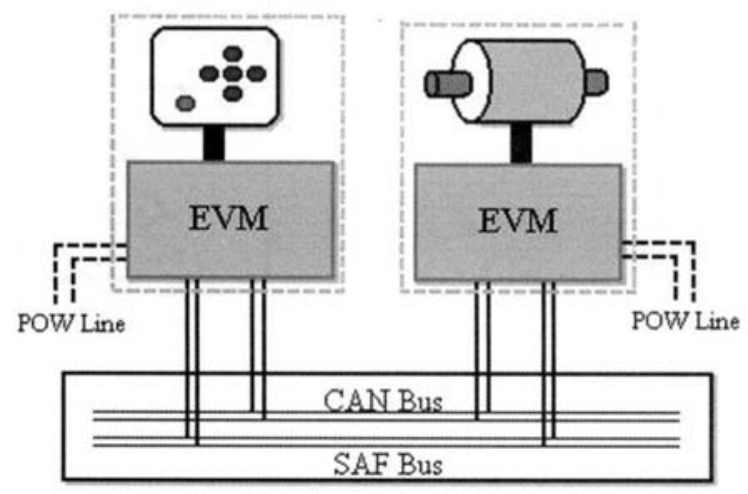

Fig.8 the diagram of the M3S system

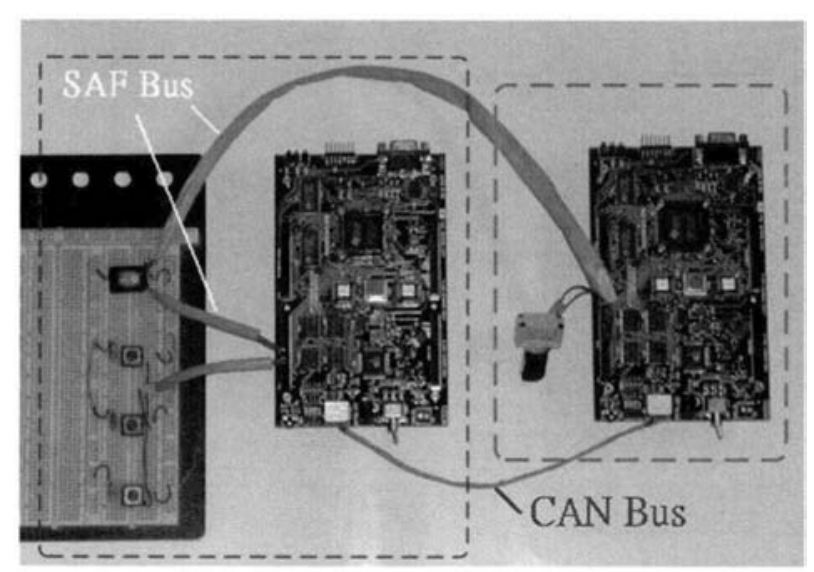

Fig.9 the photo of the M3S system 
"Left" and "Right". In order to differentiate input and output signal clearly and from overlapping and in timing, we amplify the input signal amplitude twofold.

Figure 10 shows:

$5^{\text {th }}$ second $\sim 23^{\text {rd }}$ second: we input any "forward" signal and we can see the output control signal in synchronize (figure 10 area 1 ).

$28^{\text {th }}$ second $\sim 43^{\text {rd }}$ second: we input any "left" signal and we can see the output control signal in synchronize (figure 10 area 2 ).

After $46^{\text {th }}$ second: we continuously input any "right" signal and we can see the output control signal in synchronize (figure 10 area 3 ).

At $65^{\text {th }}$ second: the input and output signals synchronously receive the "KEY Off" signals and the control signal is forced to stop transmitting (figure 10 area KEY Off).

Figure 11 is one of the three set input and output (I/O) channel control signals captured by the oscilloscope. The $x$-axis represents the time, one unit to one second. The y-axis shows the signal amplitude, one unit to 5 volt. Keying in an arbitrary control signal on the input side, the signal captured by the

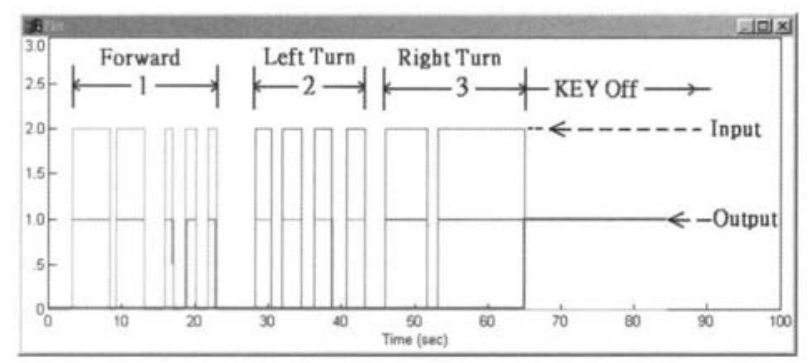

Fig.10 the results of the input and output devices signals

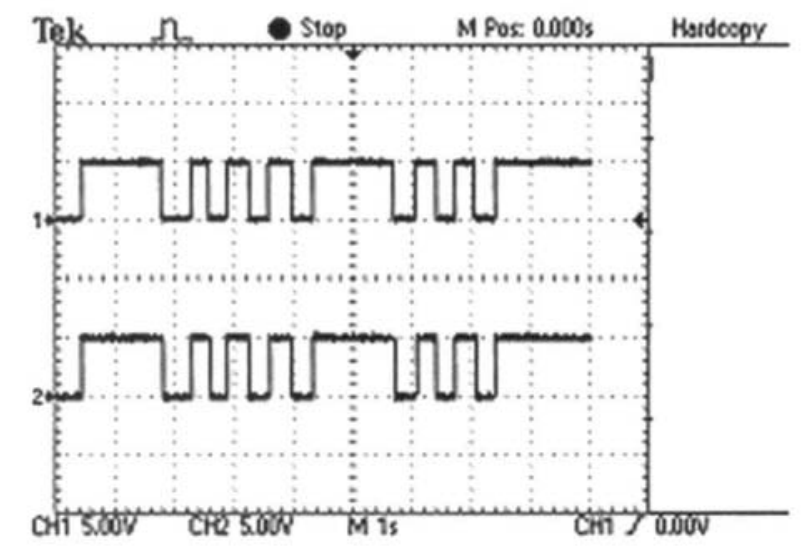

Fig.11 I/O channel control signals captured by the oscilloscope oscilloscope is shown as $\mathrm{CH} 1$ in the figure. By the test result, an arbitrary key in control signal can be successfully transmitted to the output device through the M3S bus transmission mechanism and decoded to the necessary output control signal by the digital signal processor.

\section{CONCLUSION AND DISCUSSION}

A DSP chip that has strong computing power and modulated control function is used to set up the kernel function of the intelligent M3S interface system. The transmission mechanism of the accurate control signal and the monitoring mechanism of high safe quality are included. We have tested and verified the feasibility of the intelligent M3S system used DSP to realize the M3S fundamental framework. Therefore, we can develop the complete M3S interface system and the peripheral apparatus based on this framework.

\section{REFERENCE}

1.http://www.tno.nl/m3s/ ; M3S Reference Manual Version 2.00-revision 9 July 1997.

2.TMS320F243, TMS320F241 DSP Controllers, Texas Instruments, 2000.

3.TMS320F243/F241/C242 DSP Controllers Reference Guide System and Peripherals, Texas Instruments, 2000.

4.TMS320/C24x DSP Controllers Reference Guide CPU and Instruction Set, Texas Instruments, 1999.

5.TMS320F243 Evaluation Module Technical Reference, Spectrum Digital, 1998.

6.A System Evaluation of CAN Transceivers, Texas Instruments, March 2002.

7. SN65LBC031, SN65LBC031Q, SN75LBC031 HIGH-SPEED CONTROLLER AREA NETWORK (CAN) TRANSCEIVERS, Texas Instruments, 2000.

8.CAN Specification Version 2.0, BOSCH, 1991.

9.Understanding the CAN Controller on the TMS320C24x DSP Controller, Texas Instruments, December 1998.

10.VisSim/Embedded Controls Develop User's Guide Version 4.5, Visual Solutions Incorporated, 2002.

11.Getting Started in C and Assembly Code With the TMS320LF240x DSP, Texas Instruments, 2002. 